\title{
Systematic measurements of prompt fission $\gamma$ rays - and what they tell us about fission fragment de-excitation
}

\author{
Andreas Oberstedt ${ }^{1, *}$, Angélique Gatera ${ }^{2}$, Alf Göök ${ }^{2}$, and Stephan Oberstedt ${ }^{2}$ \\ ${ }^{1}$ Extreme Light Infrastructure - Nuclear Physics (ELI-NP) / Horia Hulubei National Institute for \\ Physics and Nuclear Engineering (IFIN-HH), 077125 Bucharest-Magurele, Romania \\ ${ }^{2}$ European Commission, DG Joint Research Centre, Directorate G - Nuclear Safety and Security, Unit \\ G.2 Standards for Nuclear Safety, Security and Safeguards, 2440 Geel, Belgium
}

\begin{abstract}
In a recent measurement of prompt $\gamma$ rays from the spontaneous fission of ${ }^{252} \mathrm{Cf}$ an experimental setup was chosen that allowed the study of angular correlations between $\gamma$ rays and the nuclei from which they were emitted. The observed angular distribution of prompt fission $\gamma$ rays was then analyzed to estimate the relative contributions from radiation of different multipolarity. Preliminary results are presented and compared to previous angular correlation measurements from different fission processes and recent results from calculations with the Monte Carlo Hauser-Feshbach code FIFRELIN.
\end{abstract}

\section{Introduction}

The energy release in nuclear fission is distributed in kinetic and excitation energy of the two fragments. The latter manifests itself in fragment deformation and intrinsic excitation energy. In an early and quite simplified picture, it was assumed that the fragments are first de-excited by the emission of neutrons until the fragments' remaining excitation energy is lower than the neutron binding energy, i.e. on average at about half of it. Only then deexcitation would continue by the emission of $\gamma$ rays, eventually reaching the ground state [1]. These $\gamma$ rays may be divided into two categories, commonly called statistical and discrete $\gamma$ rays, respectively. The first ones originate mainly from electric dipole transitions and carry away most of the remaining excitation energy of the fragments, while the latter stem mainly from electric quadrupole transitions along the Yrast line and carry away most of the angular momentum of the fragments [2]. This so-called prompt $\gamma$-ray emission is a fast process, which basically takes place within the first few nanoseconds after scission [3] and is followed by $\beta$ decay(s) and thereafter by delayed emission of neutrons and $\gamma$ rays towards the valley of $\beta$ stability. The average prompt fission $\gamma$-ray multiplicity, i.e. the average number of photons per fission, amounts typically to eight [4], as corroborated by our previous measurements [5-10]. An overview on our past and future activities within this field of research is given in Ref. [11].

In this work we have chosen an experimental setup that allowed measuring angular correlations between fission fragments and prompt $\gamma$ rays, in order to study the de-excitation of the

\footnotetext{
*e-mail: andreas.oberstedt@eli-np.ro
} 


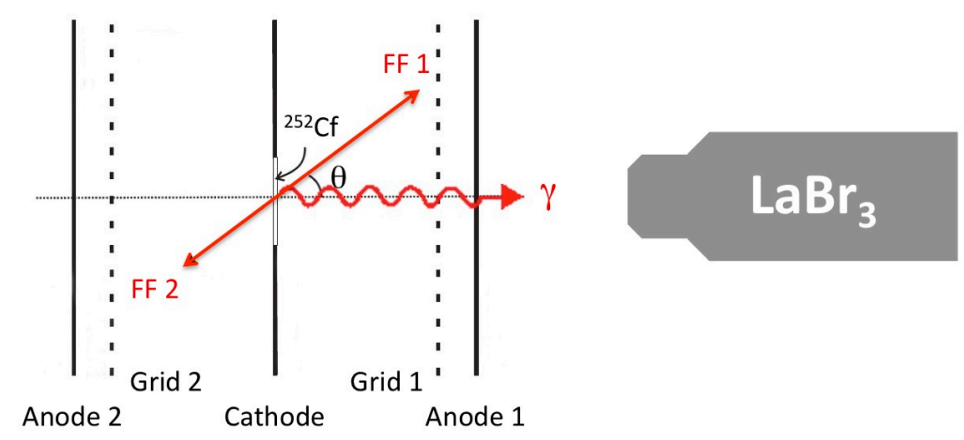

Figure 1. Sketch of the experimental setup used to measure angular correlations between fission fragments (FF 1 or FF 2) and prompt fission $\gamma$ rays. The fragments were detected with a cylindrical twin Frisch-grid ionization chamber (FGIC) on whose central cathode a ${ }^{252} \mathrm{Cf}$ sample was mounted. For the detection of the $\gamma$ rays a $\mathrm{LaBr}_{3}$ :Ce detector of size $76 \mathrm{~mm} \times 76 \mathrm{~mm}$ was employed that was placed along the symmetry axis perpendicular to the sample. The polar angle of the emitted fragments equivalent to the angle relative to the detected photons is denoted by $\theta$.

fragments by prompt $\gamma$-ray emission and to deduce information about the relative contributions from radiation of different multipolarity. Below we compare our observations to those from previous angular correlation measurements and give first preliminary results, which are discussed in terms of results from recent calculations with the Monte Carlo Hauser-Feshbach code FIFRELIN [12].

\section{Experimental setup and data treatment}

The recent measurement of PFGS from ${ }^{252} \mathrm{Cf}(\mathrm{sf})$ was performed with a $76 \mathrm{~mm} \times 76 \mathrm{~mm}$ (diameter $\times$ length) $\mathrm{LaBr}_{3}:$ Ce scintillation detector that was placed perpendicular to the plane of the sample along its symmetry axis. The ${ }^{252} \mathrm{Cf}$ sample was mounted inside a cylindrical twin Frisch-grid ionization chamber (FGIC) [13] in the center of a common cathode. Fission fragments from a thin ${ }^{252} \mathrm{Cf}$ sample are emitted to both sides of the cathode, ionizing the P-10 counting gas, and being detected by means of the electrons moving towards the respective anodes. A Frisch grid is placed in front of each anode in order to shield the latter from the influence of the ions, but it also allows determining the (polar) emission angle of the fragments (see Fig. 1 for a schematic view of the experimental setup). The FGIC provides the fission trigger for the coincident measurement of $\gamma$ rays, for which energy and time-offlight (TOF) between sample and detector are recorded. The latter information is used to distinguish prompt $\gamma$ rays from other photons, emitted e.g. in prompt fission neutron induced reactions, i.e. mainly inelastic neutron scattering. The experimental techniques applied here are in principle the same as described in Refs. [5-10], where more details may be found.

The collected prompt fission $\gamma$ rays were sorted into energy spectra for $\cos \theta$ bins between 0 and 1 in steps of 0.05 . In order to obtain emission spectra, the response function of the detector has to be determined, which is usually done by means of Monte Carlo simulations of the actual setup with computer codes like e.g. Penelope [14] or Geant4 [15]. Thereafter the response function must be unfolded from the raw spectra. However, as a first step, we have chosen here a different, less time-consuming approach. Adding up all spectra gives an angular integrated raw spectrum that may be compared with others, obtained previously for the same fissioning system. Since they all turned out to exhibit a very similar appearance, we may 


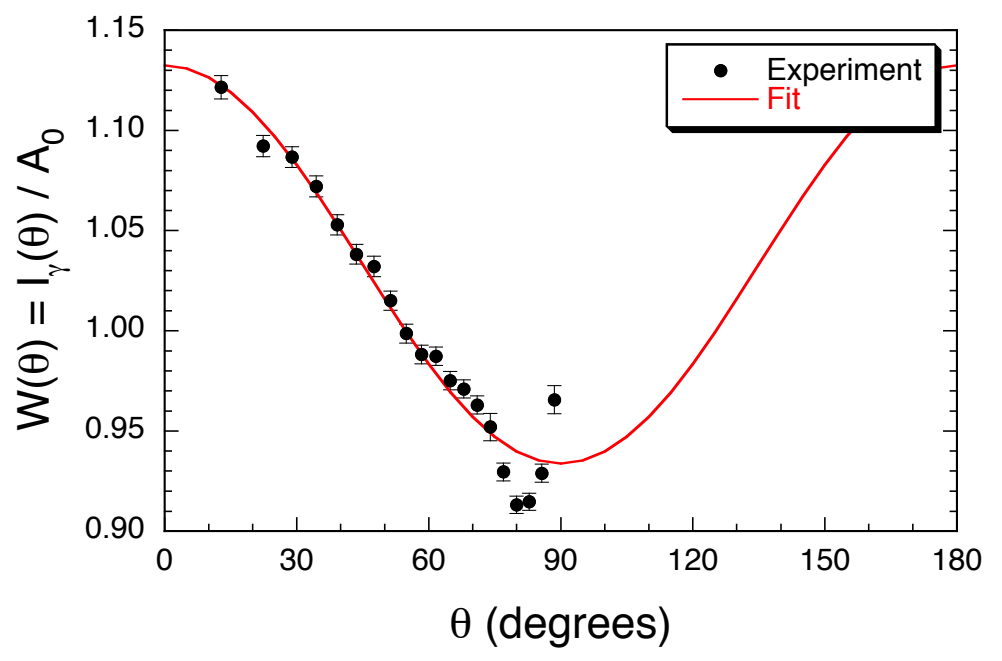

Figure 2. Measured angular distribution of prompt $\gamma$ rays from ${ }^{252} \mathrm{Cf}(\mathrm{sf})$, depicted as black dots. The error bars correspond to statistical uncertainties only. The line represents the result of a fit according to Eq. 1. The obtained coefficients are $\left\{A_{2} / A_{0}\right\}=0.13 \pm 0.03$ and $\left\{A_{4} / A_{0}\right\}=-0.0092 \pm 0.0086$, with $A_{0}=$ $0.930 \pm 0.005$.

benefit from the fact that similar raw spectra lead to similar emission spectra, provided that detector and setup are comparable [7]. This is the case here. From a previous measurement [5], for which the proper unfolding was carried out, both measured and emission spectra are known, whose ratio gives a transformation function (see Ref. [7] for details). Multiplying this function with the recently measured PFGS gives then the emission spectrum. In the same way angular-dependent emission spectra were created for each bin, from which PFGS characteristics were determined. A detailed inspection of the obtained angular distribution is performed below.

\section{Angular distribution of prompt fission $\gamma$ rays}

As described in the previous chapter, emission spectra of prompt fission $\gamma$ rays were created for $\cos \theta$ bins, covering the polar angle range $0^{\circ}<\theta<90^{\circ}$. The integrated total multiplicity, i.e. the average number of emitted photons per fission, was determined to $\bar{M}_{\gamma}=8.28 \pm 0.51$, where the statistical uncertainty is 0.07 and the systematic one, mainly due to the transformation function, amounts to 0.44 . This result is in good agreement with our last published value $\bar{M}_{\gamma}=8.29 \pm 0.13$ [6]. The angular distribution of radiation may be expressed according to

$$
I_{\gamma}(\theta)=A_{0}\left[1+\left\{A_{2} / A_{0}\right\} P_{2}(\cos \theta)+\left\{A_{4} / A_{0}\right\} P_{4}(\cos \theta)\right],
$$

where $P_{k}(\cos \theta)$ denote Legendre polynomials for $\mathrm{k}=2,4$ and $\theta$ is the emission angle of the fission fragment relative to the $\gamma$ ray direction, i.e. perpendicular to the cathode plane of the FGIC. Any possible attenuation coefficients are assumed to be 1 . The coefficients $\left\{A_{k} / A_{0}\right\}$ are determined experimentally and may be compared to theory for different types of radiation. For instance, $\left\{A_{2} / A_{0}\right\} \approx-0.3$ for pure dipole radiation and $\left\{A_{2} / A_{0}\right\} \approx+0.3$ for pure quadrupole radiation, while $\left\{A_{4} / A_{0}\right\}$ is close to zero [16]. Figure 2 shows the measured angular distribution as black dots, together with the result of a fit according to the expression given 


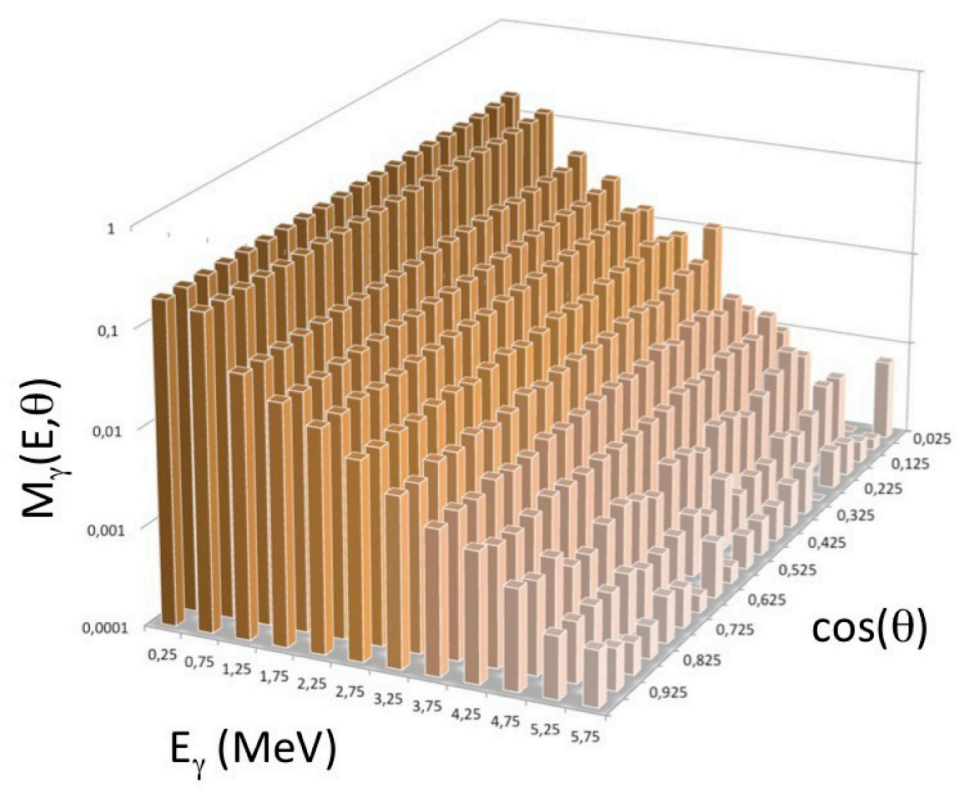

Figure 3. Three-dimensional presentation of measured PFGS from ${ }^{252} \mathrm{Cf}(\mathrm{sf})$ for angular bins of $\Delta \cos \theta$ $=0.05$ and energy bins of $\Delta \mathrm{E}=500 \mathrm{keV}$.

in Eq. 1 shown as line. It should be noted that the data close to both $\theta=0^{\circ}$ and $90^{\circ}$ suffers from considerable instrumental errors due to absorption and scattering, but these points were taken into account anyway, since they did not influence much the fit result. The obtained coefficient is $\left\{A_{2} / A_{0}\right\}=0.13 \pm 0.03$, which may be considered the result of a superposition of dipole and quadrupole photons (higher multipolarities are less probable and neglected here). Accordingly, it follows that $(+0.3) \times p+(-0.3) \times(1-p)=0.13$, with $p$ denoting the probability for quadrupole radiation. This leads to $p=0.72$, corresponding to $72 \%$ quadrupole $(\mathrm{L}=2)$ and $28 \%$ dipole $(\mathrm{L}=1)$ radiation.

In the next step we tried to assess how the ratio between quadrupole and dipole radiation depends on the energy of the prompt fission $\gamma$ rays. For that reason the PFGS was rebinned into $500 \mathrm{keV}$ intervals (see Fig. 3), for which the corresponding angular distributions were fitted according to Eq. 1 as described above for the entire spectrum. As a consequence, the coefficient $\left\{A_{2} / A_{0}\right\}$ was determined for each energy bin and the contributions for $\mathrm{L}=1$ and $\mathrm{L}=2$ deduced. The resulting multipolarity-dependent spectra are shown on the left-hand side of Fig. 4. These results are discussed in the next section.

\section{Discussion and outlook}

Prior to this work, angular distributions of prompt $\gamma$ rays were measured in thermal-neutron induced fission of the target nuclei ${ }^{233} \mathrm{U},{ }^{235} \mathrm{U}$ and ${ }^{239} \mathrm{Pu}$ [17], as well as the spontaneous fission of ${ }^{252} \mathrm{Cf}$ [18], however the latter one only for $\gamma$ rays between 0.1 and $1.5 \mathrm{MeV}$. A comparison of the coefficients in Eq. 1 from this work and from Ref. [18] for the region $\mathrm{E}_{\gamma}=0.1$ - 1.5 MeV exhibits some difference, since we obtain $\left\{A_{2} / A_{0}\right\}=0.134$ and $\left\{A_{4} / A_{0}\right\}=0.002$, while corresponding values of 0.0683(1) and -0.0308(2), respectively, are reported in Ref. [18]. Anyway, a qualitative comparison of all these measurements exhibits a good agree- 

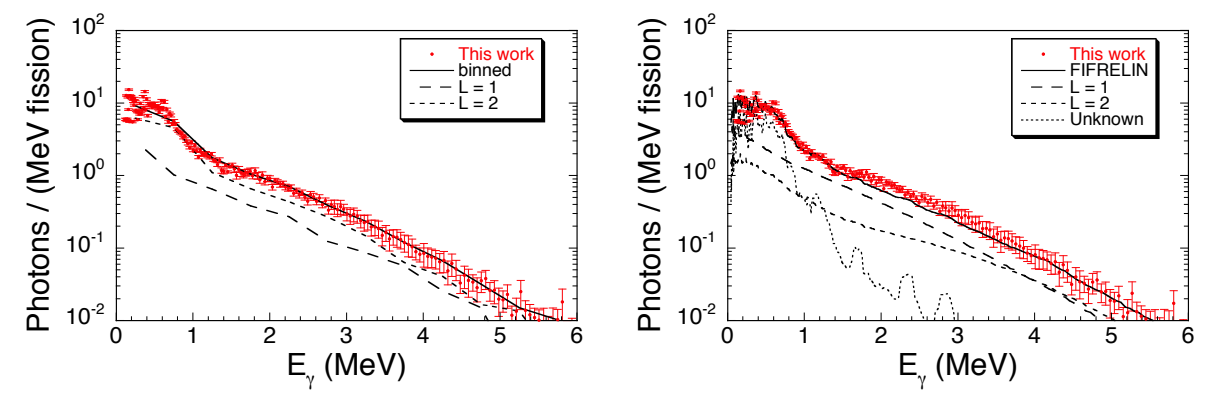

Figure 4. Measured PFGS for ${ }^{252} \mathrm{Cf}(\mathrm{sf})$ from this work, depicted by red dots. In the left part it is compared to the rebinned spectrum of $500 \mathrm{keV}$ intervals (full black line), from which the contributions for dipole $(\mathrm{L}=1)$ and quadrupole $(\mathrm{L}=2)$ radiation were determined (long and short dashed lines, respectively). The right part shows in addition to the measured spectrum a spectrum and its contributions for $\mathrm{L}=1$ and $\mathrm{L}=2$ as well as a contribution for which the multipolarity is unknown (all three as black lines), calculated recently with FIFRELIN (see text for details).

ment, indicating a dominating contribution of quadrupole radiation, of which E2 is much more likely than M2. If this E2 radiation is assigned to $\gamma$ decays along the Yrast line (cf. Sect. 1), the corresponding multipolarity-dependent multiplicity would be $\bar{M}_{\gamma, L=2} \approx 6.0$, with a total average multiplicity in excellent agreement with $\bar{M}_{\gamma}=8.29 \pm 0.13$ [6]. Each quadrupole photon will then carry away $2 \hbar$, which implies an average angular momentum of $\mathbf{J} \approx 12 \hbar$, which is in rather good agreement with $\mathbf{J} \approx 10 \hbar$ reported earlier [19] and results from GEF calculations for different fission fragments [20]. It has to be mentioned though that also lower fission fragment spins of $6 \hbar$ to $8 \hbar$ have been reported (see [18] and references therein). Hence, further experimental studies are necessary. The remaining dipole multiplicity would then be $\bar{M}_{\gamma, L=1} \approx 2.3$ for statistical $\gamma$ rays.

Calculations based on the Monte Carlo Hauser-Feshbach code FIFRELIN [12] have previously been rather successful in reproducing experimental results for PFGS characteristics as well as the distinct peak structure of the low-energy part of the energy spectra for ${ }^{235} \mathrm{U}\left(\mathrm{n}_{t h}\right.$, f) [8] and ${ }^{252} \mathrm{Cf}(\mathrm{sf})$ [6]. For comparison, the calculated average PFGS multiplicity has been adjusted to the measured one. Even multipolarity-dependent spectra were generated [21], which however seem to show a dominating contribution of $\mathrm{L}=1$ radiation. Apparently, this is in contrast with the experimental results presented in this work. New FIFRELIN calculations were performed recently [22], whose results are shown on the right-hand side of Fig. 4. However, it must be noted here that, apart from $\gamma$ rays of known multipolarity 1 and 2 , there is a considerable amount of photons, whose multipolarity is not assigned (denoted with "unknown" in the figure), since they correspond to transitions between levels taken from the RIPL-3 database [23]. In fact, they constitute about $44 \%$ of the calculated total average PFGS multiplicity, as shown in Table 1. Since a dominating contribution of quadrupole radiation has been observed in all PFGS measurements, as referred to above, we tend to conclude that most of these transitions of unknown multipolarity should lead to quadrupole radiation. Therefore, the sum of these "unknown" transitions and the calculated ones with $\mathrm{L}=2$ should be compared to the experimentally obtained contribution for $\mathrm{L}=2$. With this condition comparison of the values given Table 1 shows indeed a rather good agreement.

This agreement would have been even better, if a probably more realistic assumption for the coefficient for pure dipole radiation, $\left\{A_{2} / A_{0}\right\} \approx-0.2$, had been made, considering the value $\left\{A_{2} / A_{0}\right\}=-0.212(26)$ from the example given in Ref. [16]. With this value, $\bar{M}_{\gamma, L=1} \approx$ 
Table 1. Prompt $\gamma$-ray characteristics for the spontaneous fission of ${ }^{252} \mathrm{Cf}$. Experimental results from this work for the average $\gamma$-ray multiplicity $\bar{M}_{\gamma}$, the average energy per photon $\epsilon_{\gamma}$, and the total energy $E_{\gamma, t o t}$ released in fission, are given for the integral spectrum as well as for the multipolarity-dependent spectra and compared to corresponding values recently calculated with FIFRELIN [22] (see text for details). Relative multiplicities are also given, under the assumption that higher multipolarities may be neglected.

\begin{tabular}{|c|c|c|c|c|}
\hline & Multipolarity L & $\begin{array}{l}\text { Exp. transitions } \\
\text { (this work) }\end{array}$ & \multicolumn{2}{|c|}{$\begin{array}{l}\text { Calculations } \\
\text { (FIFRELIN) }\end{array}$} \\
\hline $\bar{M}_{\gamma}$ & all & $8.28 \pm 0.51$ & 8.28 & (adjusted) \\
\hline $\bar{M}_{\gamma}$ & 1 & $(28 \%)$ & 3.20 & $(39 \%)$ \\
\hline $\bar{M}_{\gamma}$ & 2 & $(72 \%)$ & 1.45 & $(17 \%)$ \\
\hline $\bar{M}_{\gamma}$ & experimental & & 3.63 & $(44 \%)$ \\
\hline$\epsilon_{\gamma}$ & all & $0.79 \pm 0.10 \mathrm{MeV}$ & $0.76 \mathrm{I}$ & \\
\hline$\epsilon_{\gamma}$ & 1 & $0.86 \mathrm{MeV}$ & $0.94 \mathrm{I}$ & \\
\hline$\epsilon_{\gamma}$ & 2 & $0.76 \mathrm{MeV}$ & $1.03 \mathrm{I}$ & \\
\hline$\epsilon_{\gamma}$ & experimental & & $0.50 \mathrm{I}$ & \\
\hline $\mathrm{E}_{\gamma, t o t}$ & all & $6.51 \pm 0.76 \mathrm{MeV}$ & $6.30 \mathrm{I}$ & \\
\hline $\mathrm{E}_{\gamma, t o t}$ & 1 & $1.99 \mathrm{MeV}$ & $3.00 \mathrm{~N}$ & \\
\hline $\mathrm{E}_{\gamma, t o t}$ & 2 & $4.52 \mathrm{MeV}$ & $1.49 \mathrm{I}$ & \\
\hline $\mathrm{E}_{\gamma, t o t}$ & experimental & & $1.81 \mathrm{I}$ & \\
\hline
\end{tabular}

2.8 and $\bar{M}_{\gamma, L=2} \approx 5.5$ would have been obtained, corresponding to relative contributions of $34 \%$ and $66 \%$, respectively. Of course, these results are still considered preliminary and must be compared with other related information on fission fragments, such as average neutron separation energies and average moments of inertia, in order to verify the deduced energies carried away by dipole and quadrupole photons during the de-excitation process of fission fragments. Hence, these activities are to be continued.

\section{Acknowledgments}

One of the authors (A. O.) acknowledges the support from the Extreme Light Infrastructure Nuclear Physics (ELI-NP) Phase II, a project co-financed by the Romanian Government and the European Union through the European Regional Development Fund - the Competitiveness Operational Programme (1/07.07.2016, COP, ID 1334), with which this work had been finalized.

\section{References}

[1] C. Wagemans (editor), The Nuclear Fission Process, CRC Press Boca Raton (1991).

[2] A. Hotzel, P. Thirolf, Ch. Ender, D. Schwalm, M. Mutterer, P. Singer, M. Klemens, J. P. Theobald, M. Hesse, F. Gönnenwein, H. v. d. Ploeg, Z. Phys. A 356, 299 (1996).

[3] P. Talou, T. Kawano, I. Stetcu, J. P. Lestone, E. McKigney, and M. B. Chadwick, Phys. Rev. C94, 064613 (2016).

[4] R. Vandenbosch and J.R. Huizenga, Nuclear Fission, Academic Press New York and London (1973).

[5] R. Billnert, F.-J. Hambsch, A. Oberstedt, and S. Oberstedt, Phys. Rev. C87, 024601 (2013). 
[6] A. Oberstedt, R. Billnert, F.-J. Hambsch, and S. Oberstedt, Phys. Rev. C92, 014618 (2015).

[7] S. Oberstedt, A. Oberstedt, A. Gatera, A. Göök, F.-J. Hambsch, A. Moens, G. Sibbens, D. Vanleeuw, and M. Vidali, Phys. Rev. C93, 054603 (2016).

[8] A. Oberstedt, T. Belgya, R. Billnert, R. Borcea, T. Bryś, W. Geerts, A. Göök, F.-J. Hambsch, Z. Kis, T. Martinez, S. Oberstedt, L. Szentmiklosi, K. Takács, and M. Vidali, Phys. Rev. C87, 051602(R) (2013).

[9] S. Oberstedt, R. Billnert, T. Belgya, T. Bryś, W. Geerts, C. Guerrero, F.-J. Hambsch, Z. Kis, A. Moens, A. Oberstedt, G. Sibbens, L. Szentmiklosi, D. Vanleeuw, and M. Vidali, Phys. Rev. C90, 024618 (2014).

[10] A. Gatera, T. Belgya, A. Göök, F.-J. Hambsch, M. Lebois, A. Oberstedt, S. Oberstedt, L. Qi, L. Szentmiklósi, G. Sibbens, and M. Vidali, Phys. Rev. C95, 064609 (2017).

[11] S. Oberstedt, R. Billnert, F.-J. Hambsch, M. Lebois, A. Oberstedt, and J.N. Wilson, Eur. Phys. J. A 51 (2015) 178.

[12] O. Litaize and O. Serot, Phys. Rev. C82, 054616 (2010).

[13] C. Budtz-Jørgensen, H.-H. Knitter, Ch. Straede, F.-J. Hambsch and R. Vogt, Nucl. Instr. and Meth. A 258, 209 (1987).

[14] http://www.oecd-nea.org/tools/abstract/detail/nea-1525.

[15] S. Agostinelli and the Geant4 collaboration, Nucl. Inst. Meth. A 506 (2003) 250.

[16] E.S. Paul, UK Nuclear Physics Summer School, Bristol, 27. August - 6. September 2013, http://ns.ph.liv.ac.uk/ ajb/summerschool/files/ESP-Lecture4.pdf

[17] M.M. Hoffman, Phys. Rev. 133, B714 (1964).

[18] Yu. N. Kopach, P. Singer, M. Mutterer, M. Klemens, A. Hotzel, D. Schwalm, P. Thirolf, M. Hesse, and F. Gönnenwein, Phys. Rev. Lett. 82, 303 (1999).

[19] A. Bogachev, L. Krupa, O. Dorvaux, E. Kozulin, M. Itkis, M.-G. Porquet, A. Astier, D. Curien, I. Deloncle, G. Duchene, B.J.P. Gall, F. Hanappe, F. Khalfallah, M. Rousseau, L. Stuttge, N. Redon, and O. Stezowski, Eur. Phys. J. A 34, 23 (2007).

[20] K.-H. Schmidt, B. Jurado, C. Amouroux and C. Schmitt, Nuclear Data Sheets 131, 207 (2016).

[21] D. Regnier, "Contribution à l'étude des gammas prompts de fission", $\mathrm{PhD}$ thesis, Université de Grenoble, France (2013).

[22] A. Chebboubi, private communication (2017).

[23] R. Capote, M. Herman, P. Oblozinsky, P. Young, S. Goriely, T. Belgya, A. Ignatyuk, A. Koning, S. Hilaire, V. Plujko, M. Avrigeanu, O. Bersillon, M. Chadwick, T. Fukahori, Z. Ge, Y. Han, S. Kailas, J. Kopecky, V. Maslov, G. Reffo, M. Sin, E. Soukhovitskii, P. Talou, RIPL - Reference Input Parameter Library for Calculation of Nuclear Reactions and Nuclear Data Evaluations, Nucl. Data Sheets 110 (12) (2009) 3107. 